\title{
A multi-objective optimal decision model for a green closed-loop supply chain under uncertainty: A real industrial case study
}

\author{
Fang, I.W. ${ }^{a}$, Lin, W.-T. ${ }^{b}$ \\ ${ }^{\text {a }}$ National ChengChi University, Department of Management Information Systems, Taipei, Taiwan (R.O.C) \\ ${ }^{\mathrm{b}}$ National ChengChi University, Department of Management Information Systems, Taipei, Taiwan (R.O.C)
}

\begin{abstract}
A B S T R A C T
Green closed-loop supply chain management is an important topic for business operations today because of increasing resource scarcity and environmental issues. Companies not only have to meet environmental regulations, but also must ensure high quality supply chain operation as a means to secure competitive advantages and increase profits. This study proposes a multiobjective mixed integer programming model for an integrated green closedloop supply chain network designed to maximize profit, amicable production level (environmentally friendly materials and clean technology usage), and quality level. A scenario-based robust optimization method is used to deal with uncertain parameters such as the demand of new products, the return rates of returned products and the sale prices of remanufactured products. The proposed model is applied to a real industry case example of a manufacturing company to illustrate the applicability of the proposed model. The result shows a robust optimal resource allocation solution that considers multiple scenarios. This study can be a reference for closed-loop supply chain related academic research and also can be used to guide the development of a green closed-loop supply chain model for better decision making.
\end{abstract}

\author{
ARTICLE INFO \\ Keywords: \\ Green closed-loop supply chain; \\ Sustainability; \\ Modelling; \\ Robust optimization; \\ Mixed integer programming \\ model; \\ Supply chain management; \\ Uncertainty; \\ LP-metric method \\ *Corresponding author: \\ 102356508@nccu.edu.tw \\ (Fang, I.W.) \\ Article history: \\ Received 5 June 2021 \\ Revised 23 June 2021 \\ Accepted 24 June 2021

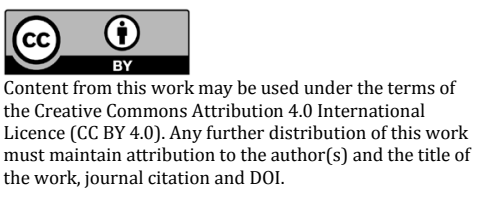

\section{References}

[1] Shi, J., Zhang, G., Sha, J. (2011). Optimal production planning for a multi-product closed loop system with uncertain demand and return, Computers \& Operations Research, Vol. 38, No. 3, 641-650, doi: 10.1016/ j.cor.2010.08.008.

[2] Jabbarzadeh, A., Haughton, M., Khosrojerdi, A. (2018). Closed-loop supply chain network design under disruption risks: A robust approach with real world application, Computers \& Industrial Engineering, Vol. 116, 178-191, doi: $10.1016 /$ j.cie.2017.12.025.

[3] Tseng, M., Lim, M., Wong, W.P. (2015). Sustainable supply chain management: A closed-loop network hierarchical approach, Industrial Management \& Data Systems, Vol. 115, No. 3, 436-461, doi: 10.1108/IMDS-10-2014$\underline{0319}$.

[4] Gu, X., Ieromonachou, P., Zhou, L., Tseng, M.-L. (2018). Optimising quantity of manufacturing and remanufacturing in an electric vehicle battery closed-loop supply chain, Industrial Management \& Data Systems, Vol. 118, No. 1, 283-302, doi: 10.1108/IMDS-04-2017-0132. 
[5] Fang, I.-W., Lin, W.-T. (2019). A robust optimization model for a green closed-loop supply chain network design, In: 2019 IEEE $6^{\text {th }}$ International Conference on Industrial Engineering and Applications (ICIEA), Waseda University, Tokyo, Japan, 723-727, doi: 10.1109/IEA.2019.8714928.

[6] Govindan, K., Soleimani, H., Kannan, D. (2015). Reverse logistics and closed-loop supply chain: A comprehensive review to explore the future, European Journal of Operational Research, Vol. 240, No. 3, 603-626, doi: 10.1016/j.ejor.2014.07.012.

[7] Žic, J., Žic, S. (2020). Multi-criteria decision making in supply chain management based on inventory levels, environmental impact and costs, Advances in Production Engineering \& Management, Vol. 15, No. 2, 151-163, doi: 10.14743/apem2020.2.355.

[8] Zhao, R., Liu, Y., Zhang, N., Huang, T. (2017). An optimization model for green supply chain management by using a big data analytic approach, Journal of Cleaner Production, Vol. 142, Part 2, 1085-1097, doi: 10.1016/i.jclepro. 2016.03.006.

[9] Talaei, M., Moghaddam, B.F., Pishvaee, M.S., Bozorgi-Amiri, A., Gholamnejad, S. (2016). A robust fuzzy optimization model for carbon-efficient closed-loop supply chain network design problem: A numerical illustration in electronics industry, Journal of Cleaner Production, Vol. 113, 662-673, doi: 10.1016/j.jclepro.2015.10.074.

[10] Ma, R., Yao, L., Jin, M., Ren, P., Lv, Z. (2016). Robust environmental closed-loop supply chain design under uncertainty, Chaos, Solitons \& Fractals, Vol. 89, 195-202, doi: 10.1016/j.chaos.2015.10.028.

[11] Altmann, M., Bogaschewsky, R. (2014). An environmentally conscious robust closed-loop supply chain design, Journal of Business Economics, Vol. 84, 613-637, doi: 10.1007/s11573-014-0726-4.

[12] Amin, S.H., Zhang, G. (2013). A multi-objective facility location model for closed-loop supply chain network under uncertain demand and return, Applied Mathematical Modelling, Vol. 37, No. 6, 4165-4176, doi: 10.1016/ j.apm.2012.09.039.

[13] Liu, M., Liu, R., Zhu, Z., Chu, C., Man, X. (2018). A bi-objective green closed loop supply chain design problem with uncertain demand, Sustainability, Vol. 10, No. 4, 967-988, doi: 10.3390/su10040967.

[14] Das, K., Posinasetti, N.R. (2015). Addressing environmental concerns in closed loop supply chain design and planning, International Journal of Production Economics, Vol. 163, 34-47, doi: 10.1016/i.ijpe.2015.02.012.

[15] Saffar, M.M., Shakouri, H.G., Razmi, J. (2015). A new multi objective optimization model for designing a green supply chain network under uncertainty, International Journal of Industrial Engineering Computations, Vol. 6, 1532, doi: $10.5267 /$ i.ijiec.2014.10.001.

[16] Pourjavad, E., Mayorga, R.V. (2018). Optimization of a sustainable closed loop supply chain network design under uncertainty using multi-objective evolutionary algorithms, Advances in Production Engineering \& Management, Vol. 13, No. 2, 216-228, doi: 10.14743/apem2018.2.286.

[17] Franca, R.B., Jones, E.C., Richards, C.N., Carlson, J.P. (2010). Multi-objective stochastic supply chain modeling to evaluate tradeoffs between profit and quality, International Journal Production Economics, Vol. 127, No. 2, 292299, doi: 10.1016/j.ijpe.2009.09.005.

[18] Ramezani, M., Bashiri, M., Tavakkoli-Moghaddam, R. (2013). A new multi-objective stochastic model for a forward/reverse logistic network design with responsiveness and quality level, Applied Mathematical Modelling, Vol. 37, No. 1-2, 328-344, doi: 10.1016/j.apm.2012.02.032.

[19] Gharaei, A., Karimi, M., Hoseini Shekarabi, S.A. (2019). An integrated multi-product, multi-buyer supply chain under penalty, green, and quality control polices and a vendor managed inventory with consignment stock agreement: The outer approximation with equality relaxation and augmented penalty algorithm, Applied Mathematical Modelling, Vol. 69, 223-254, doi: 10.1016/j.apm.2018.11.035.

[20] Jafari Ashlaghi, M. (2014). A new approach to green supplier selection based on fuzzy multi-criteria decision making method and linear physical programming, Tehnički Vjesnik - Technical Gazette, Vol. 21, No. 3, 591-597.

[21] Mohammed, F., Selim, S.Z., Hassan, A., Syed, M.N. (2017). Multi-period planning of closed-loop supply chain with carbon policies under uncertainty, Transportation Research Part D: Transport and Environment, Vol. 51, 146-172, doi: 10.1016/j.trd.2016.10.033.

[22] Karimi, B., Niknamfar, A.H., Gavyar, B.H., Barzegar, M., Mohtashami, A. (2019). Multi-objective multi-facility green manufacturing closed-loop supply chain under uncertain environment, Assembly Automation, Vol. 39, No. 1, 58-76, doi: 10.1108/AA-09-2018-0138.

[23] Valizadeh, J., Sadeh, E., Sabegh, Z.A., Hafezalkotob, A. (2020). Robust optimization model for sustainable supply chain for production and distribution of polyethylene pipe, Journal of Modelling in Management, Vol. 15 No. 4, 1613-1653, doi: 10.1108/JM2-06-2019-0139.

[24] Alem, D.J., Morabito, R. (2012). Production planning in furniture settings via robust optimization, Computers \& Operations Research, Vol. 39, No. 2, 139-150, doi: 10.1016/j.cor.2011.02.022.

[25] Mulvey, J.M., Vanderbei, R.J., Zenios, S.A. (1995). Robust optimization of large-scale systems, Operations Research, Vol. 43, No. 2, 264-281, doi: 10.1287/opre.43.2.264.

[26] Yu, C.-S., Li, H.-L. (2000). A robust optimization model for stochastic logistic problems, International Journal of Production Economics, Vol. 64, No. 1-3, 385-397, doi: 10.1016/S0925-5273(99)00074-2.

[27] Oršič, J., Rosi, B., Jereb, B. (2019). Measuring sustainable performance among logistic service providers in supply chains, Tehnički Vjesnik - Technical Gazette, Vol. 26, No. 5, 1478-1485, doi: 10.17559/TV-20180607112607. 


\title{
Večkriterijski model za optimalno odločanje za zeleno krožno dobavno verigo $z$ negotovostmi: Industrijska študija primera
}

\author{
Fang, I.W. ${ }^{a}$, Lin, W.-T. ${ }^{b}$ \\ aNational ChengChi University, Department of Management Information Systems, Taipei, Taiwan (R.O.C) \\ bNational ChengChi University, Department of Management Information Systems, Taipei, Taiwan (R.O.C)
}

\section{POVZETEK}

Upravljanje zelene krožne dobavne verige je, zaradi vedno večjega pomanjkanja virov in okoljskih težav pomembna tema za današnje poslovanje. Podjetja morajo izpolnjevati okoljske predpise in hkrati zagotavljati visokokakovostno delovanje dobavne verige, kar jim zagotavlja konkurenčno prednost in povečanje dobička. Ta študija predlaga večkriterijski mešani celoštevilski model linearnega programiranja za integrirano omrežje zelene krožne dobavne verige, zasnovan za maksimiranje dobička, ravni sprejemljive proizvodnje (uporaba okolju prijaznih materialov in čiste tehnologije) in ravni kakovosti. Na scenariju temelječa metoda robustne optimizacije je bila uporabljena za obravnavanje negotovih parametrov, kot so povpraševanje po novih izdelkih, stopnja vračila izdelkov in prodajna cena predelanih izdelkov. Predlagani model je uporabljen na primeru resničnega proizvodnega podjetja, s čimer se ponazori uporabnost predlaganega modela. Rezultat prikazuje robustno optimalno rešitev za dodeljevanje virov, ki upošteva več scenarijev. Ta študija je lahko referenca za akademske raziskave, povezane s krožno dobavno verigo, lahko pa se uporablja tudi kot vodilo razvoja zelene krožne dobavne verige za boljše odločanje.

\section{PODATKI O ČLANKU}

Ključne besede:

Zelena krožna dobavna veriga;

Trajnost;

Modeliranje;

Robustna optimizacija;

Mešani celoštevilski model linear-

nega programiranja;

Upravljanje dobavne verige;

Negotovost;

Metoda LP-metrike

* Kontaktna oseba:

102356508@nccu.edu.tw

(Fang, I.W.)

Zgodovina članka:

Prejet 5. junija 2021

Popravljen 23. junija 2021

Sprejet 24. junija 2021

(i)

Content from this work may be used under the terms of the Creative Commons Attribution 4.0 International Licence (CC BY 4.0). Any further distribution of this work must maintain attribution to the author(s) and the title of 